\title{
Uso de un poste y núcleo de fibra de vidrio compuesto fabricados con CAD / CAM para restaurar un diente tratado endodónticamente: reporte de caso
}

Use of a CAD / CAM-fabricated composite fiberglass post and core to restore an endodontically treated tooth: case report

Henry Vilcapoma 1,a,b, Rolando Ganoza ${ }^{2 a ; 3 c}$, Anthony Bolaños 4,a, Akemy Tapia ${ }^{4, a}$, Antonio Balarezo ${ }^{2, a ; ~ 5, d ~}$

\section{RESUMEN}

En la actualidad existe un amplio uso de los postes de fibra de vidrio. Sin embargo, tienen la desventaja que su forma no se pueden cambiar y son inadecuadas para casos de dientes con amplia destrucción coronal y conductos radiculares amplios. Este reporte de caso clínico describe el uso de un poste y núcleo de fibra de vidrio fabricados con un sistema CAD/CAM para restaurar un diente premolar inferior tratado endodónticamente. Este poste de fibra de vidrio CAD/CAM se adapta mejor al conducto radicular que un poste prefabricado, sin necesidad de emplear resina compuesta para el muñón y reduce el espesor de la capa de cemento.

PALABRAS CLAVE: Técnica poste y muñón, diseño asistido por computadora, fabricación asistida por computadora, diente tratado endodónticamente.

\section{SUMMARY}

Currently, there is a wide use of fiberglass post. However, they have the disadvantage that their shape can not be changed and are inadequate for cases of teeth with extensive coronal destruction and wide root canals. This case report describes the use of a fiberglass post and core manufactured with a CAD / CAM system to restore a lower premolar tooth treated endodontically. This CAD / CAM fiberglass post adapts better to the root canal than a prefabricated post, without the need to use composite resin for the stump and reduces the thickness of the cement layer.

KEYWORDS: Post and core technic, computer aided design, computer aided manufacturing, endodontically treated tooth.

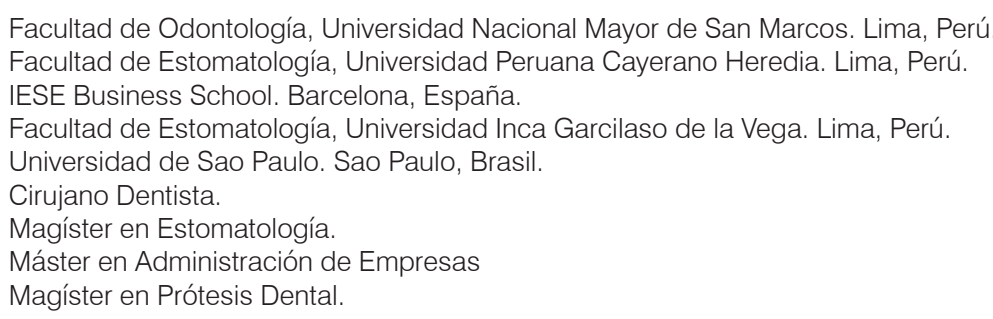




\section{INTRODUCCIÓN}

La rehabilitación oral de los dientes tratados endodónticamente con una amplia destrucción coronal requiere por lo general una restauración protésica fija retenida por un elemento de anclaje tipo poste $(1,2)$. Los postes compuestos reforzados con fibra de vidrio (postes de fibra de vidrio) son muy usados por sus buenas propiedades mecánicas, biocompatibilidad, estética y elasticidad cercana a la dentina; lo que permite distribuir de manera uniforme la tensión dentro de la raíz, disminuir el riesgo de fractura y generar (si en caso ocurra) fracturas de tipo reparable $(3,4)$. Sin embargo, presentan ciertas desventajas como la imposibilidad de personalizar sus diámetros al diámetro del conducto radicular preparado, necesidad de un núcleo de resina compuesto separado y la débil interfase entre el núcleo de resina con el espigo de fibra que genera una mayor tasa de fallos de la restauración (5).

Los factores más importantes en la retención de los postes de fibra de vidrio es el espesor del cemento alrededor del espigo y la forma del conducto radicular $(6,7)$. Diversos estudios evidencian una disminución de la resistencia de unión entre el poste de fibra y la dentina de la raíz cuando la capa de cemento es más gruesa alrededor del espigo; es por ello que no se recomienda el empleo de un poste de fibra prefabricado en un canal radicular amplio y ovalado $(8,9)$. Para ello se han propuesto varias técnicas para fabricar postes de fibra a medida del espacio del canal radicular como: poste de fibra con tiras finas de fibra de polietileno y resina (10), "poste anatómico" usando un poste de fibra y resina compuesta (11), compactación lateral de varios postes pequeños de fibra (12) y poste de fibra personalizado con una fresa diamantada $(13,14)$.

Estudios recientes han sugerido el uso de bloques compuestos de resina reforzados con fibra de vidrio para postes de fibra fabricados usando el diseño asistido por computadora / fabricación asistida por computadora (CAD/CAM) $(15,16)$. Asimismo, se ha reportado éxito en el empleo clínico de esta técnica de confección de postes de fibra aplicado en pacientes $(17,18)$.

Para confeccionar el poste de fibra CAD/CAM, se requieren imágenes digitales del diente preparado o del patrón del poste, que pueden obtenerse mediante escaneo intraoral directo, escaneo de impresiones o escaneo de modelos de yeso (19). Sin embargo diversos estudios han demostrado una mayor precisión y confiabilidad en el escaneo indirecto (impresiones y modelos de yeso); ya que la impresión digital intraoral está sujeta a diversos factores que podrían causar una variabilidad sustancial como el movimiento del paciente, limitado espacio intraoral, humedad y el flujo de saliva (20).

El objetivo del reporte de caso clínico es describir la secuencia clínica del uso de un poste y núcleo de fibra de vidrio fabricados con un sistema CAD/CAM para restaurar un diente tratado endodónticamente con un conducto radicular amplio.

La justificación del reporte de caso clínico se sustenta en la necesidad de encontrar nuevos métodos de fabricación de postes diferentes a los prefabricados de fibra de vidrio y postes colados para rehabilitar dientes tratados endodónticamente con amplia destrucción coronal y conductos amplios considerando las desventajas de los métodos hasta ahora conocidos.

\section{Presentación del caso}

Paciente de sexo masculino de 65 años, raza mestiza, atendido en el Área de Rehabilitación Oral del Posgrado y Especialización de la Universidad Peruana Cayetano Heredia, Lima, Perú, para el cambio de un puente de tres unidades cuyos pilares son los dientes 35 y 37. Luego del examen clínico y radiográfico se diagnosticó una pulpitis irreversible del diente 35 y se indicó la necesidad de un tratamiento de endodoncia en dicha pieza (figura 1).

Luego de realizado el tratamiento de conductos, se evaluó un conducto radicular relativamente amplio y la necesidad de reconstrucción del muñón coronal tanto en ancho como en altura (figura 2). El uso de un poste de fibra de vidrio prefabricado convencional no era una opción, ya que el diámetro del poste no permitiría una buena adaptación al espacio posterior a la desobturación, y se obtendría una capa de cemento gruesa que afectaría la resistencia de la unión, generando un riesgo de fractura ante la carga oclusal. Por otro lado, el espacio faltante para completar el muñón coronal era considerable para optar por el poste prefabricado y relleno con resina (u otro material de relleno de muñones) y podría generar un 


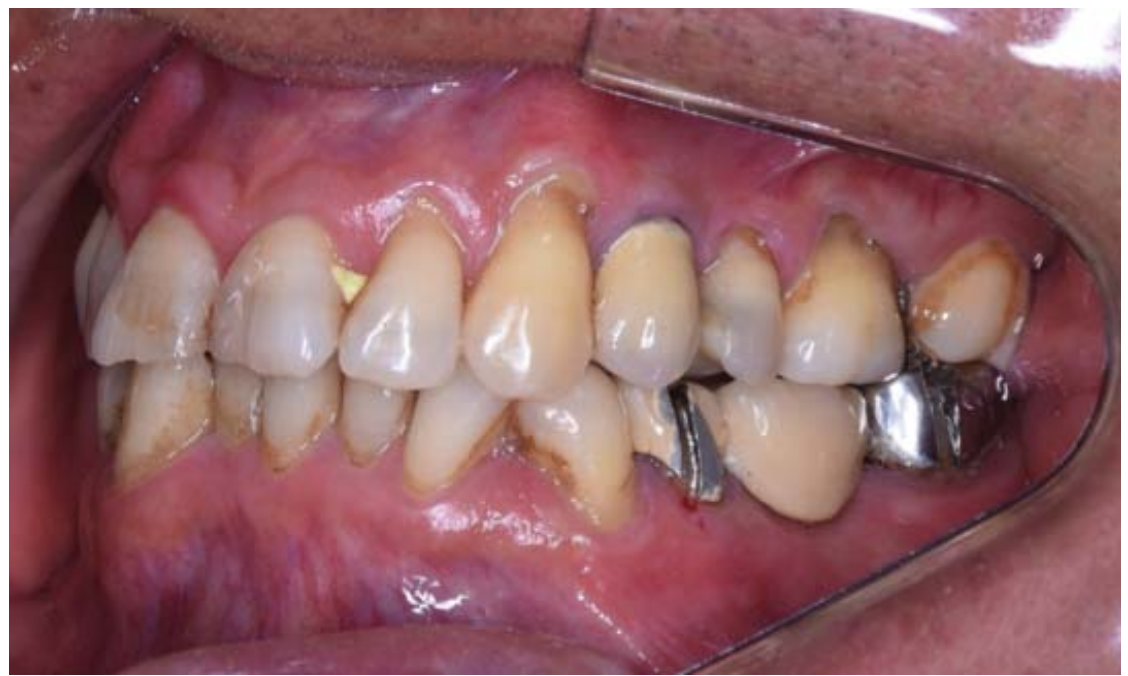

Figura 1

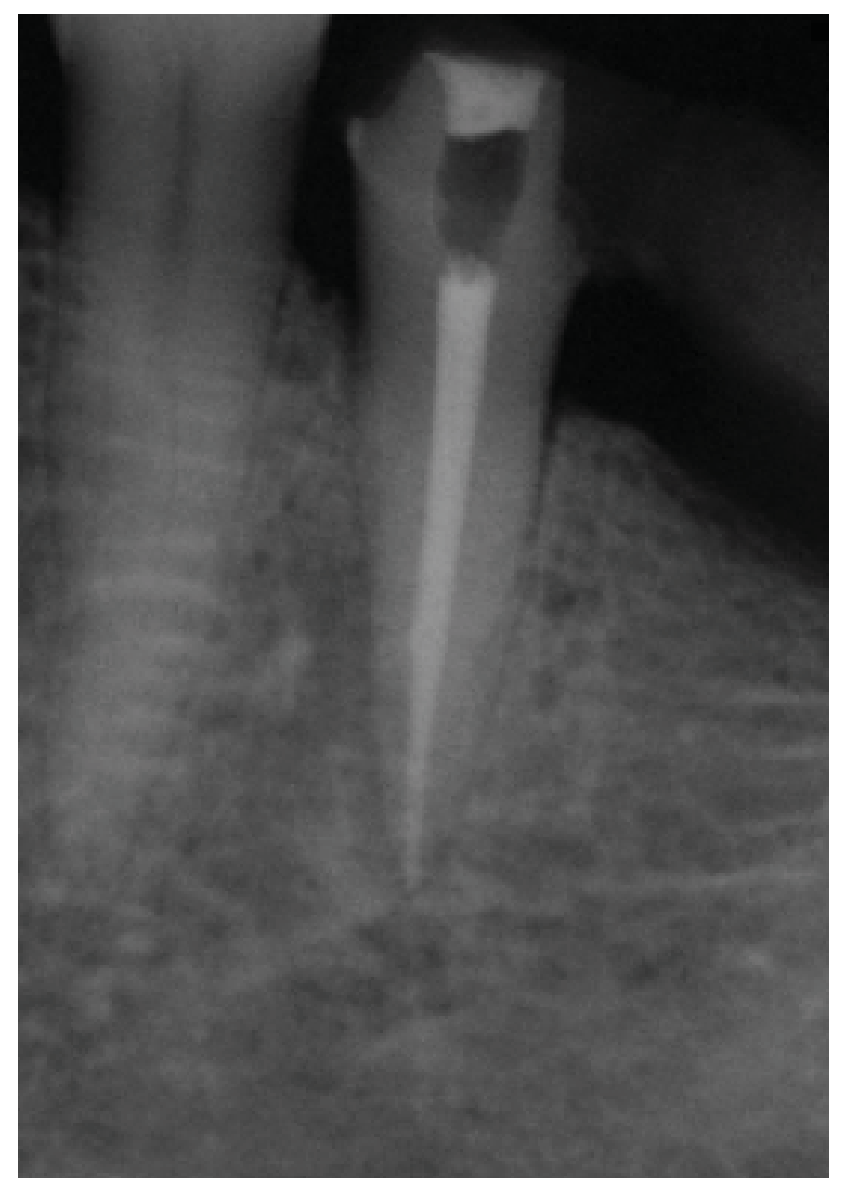

Figura 2

riego de fractura coronal ante la fuerza ejercida por ese diente como pilar de una prótesis fija.

Un poste convencional de metal fundido podría haberse utilizado para restaurar el premolar, pero considerando la rigidez y la fuerza recibida por ese premolar como pilar de prótesis fija podrían aumentar el riesgo de fractura de la raíz. Además, se consideró la negativa por parte del paciente al uso de metal y que los dientes a rehabilitar se asemejen lo más posible a los dientes naturales.

Dados estos desafíos, se planificó la confección de un espigo de fibra de vidrio fabricados con CAD/CAM y un puente de zirconio de tres unidades.

Se realizó el aislamiento absoluto del premolar y el retiro del material de obturación provisional (figura 3). El conducto radicular se preparó con instrumento de baja velocidad (Gattes y Peeso Dentsply Maillefer; Suiza). Considerando $18 \mathrm{~mm}$ como la longitud de trabajo de la endodoncia, se desobturó 13mm para la confección del espigo. Posterior a la desobturación se realizó una radiografía periapical de control para verificar la longitud de trabajo y una vez confirmado, con una lima de endodoncia \#45 (K Flexofile, Maillefer, Suiza) se procedió a eliminar los restos de gutapercha remanentes en las paredes del conducto radicular. Se procedió al lavado del conducto con cloruro de sodio al 0.9\% (Medifarma S.A, Perú) y el secado con conos de papel \#60 (Endomedic, Sure endo Corporation, Korea).

Se procedió a tomar una primera impresión con Silicona de adición en consistencia pesada (elite HD putty soft, normal set, Zhermarck, Italia) colocando una bolsa plástica encima del diente a manera de alivio para conformar una cubetilla rígida. Posterior 


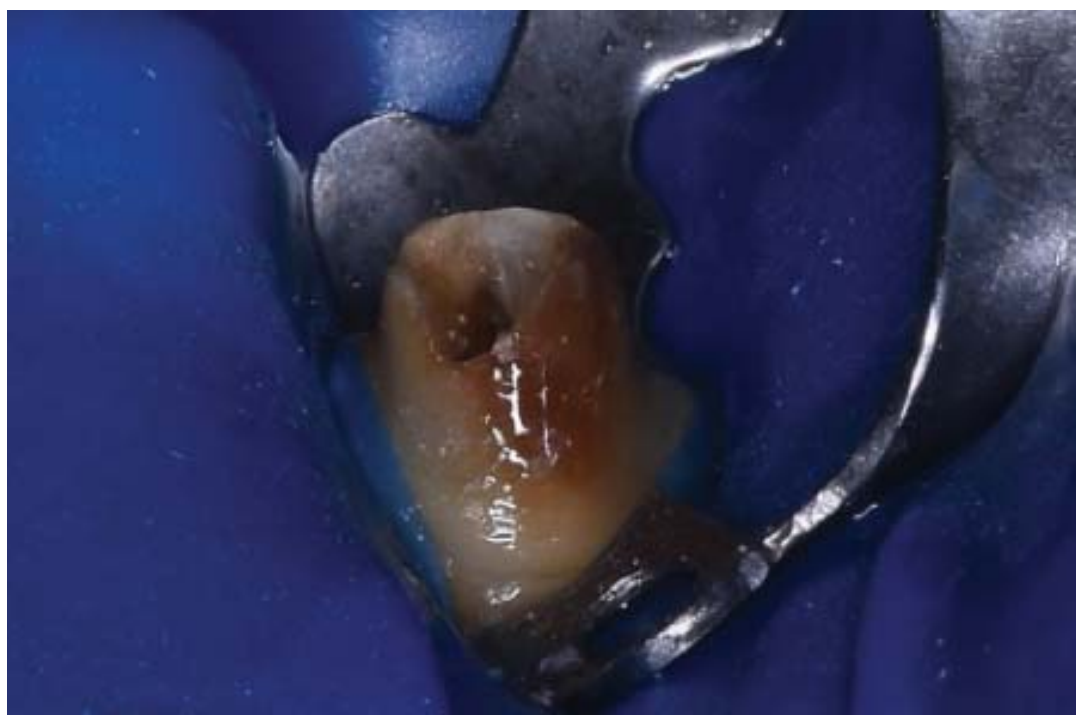

Figura 3

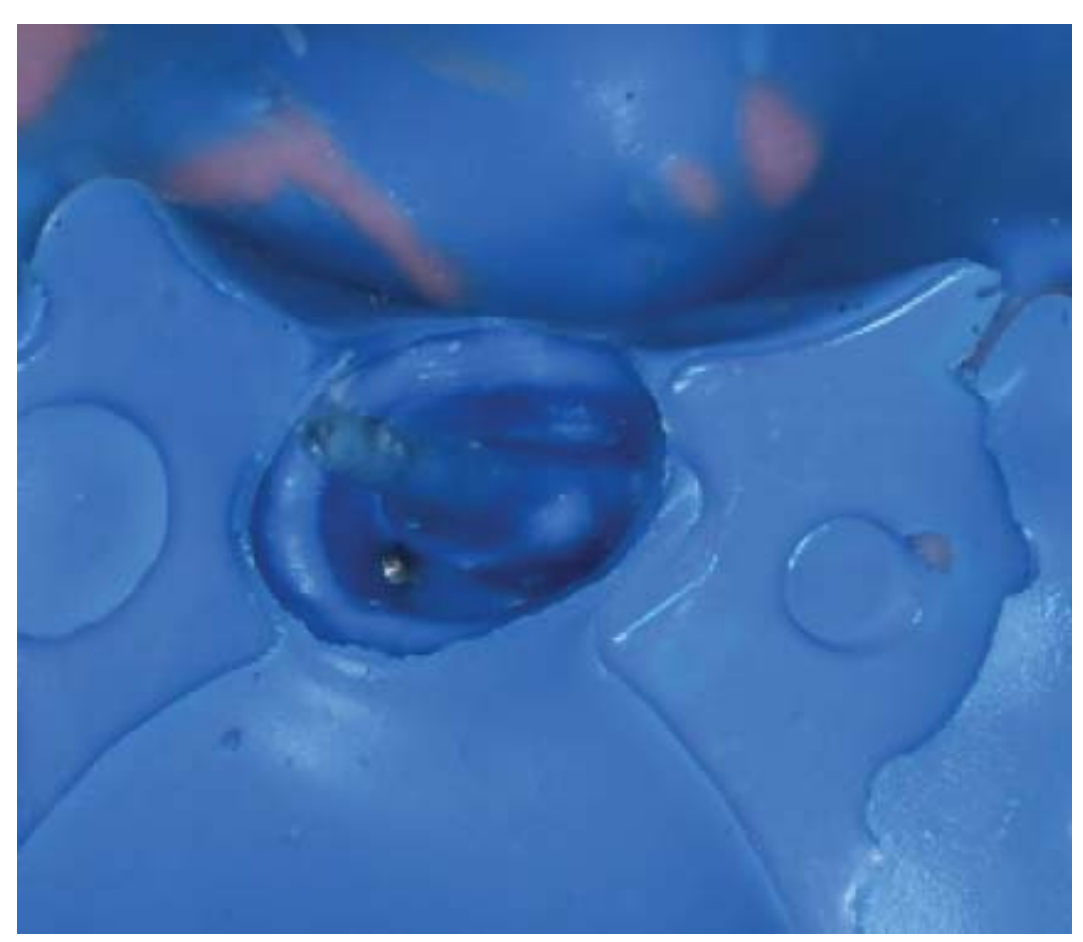

Figura 4

al retiro de la cubetilla y la bolsa plástica, se procedió a realizar una segunda impresión con silicona de adición en consistencia fluida (elite HD ligth body, Zhermarck, Italia) con la ayuda de punta de silicona y colocando un clíp metálico adaptado al conducto con adhesivo para silicona (Universal tray adhesive, Zhermarck, Italia) (figura 4).

La impresión en dos pasos fue escaneada mediante un escáner (Trios, 3SHAPE, Finlandia), obteniéndose un modelo 3D o malla (stl) que es generada por el software de CAI (Computer Aided Imaging) que en este caso fue el Design Studio (Trios, 3SHAPE, Finlandia); posterior a ello se usó dicha información del modelo 3D para el diseño del poste utilizando el software DENTAL CAD (Exocad GmbH, Darmstadt, Alemania) (figura 5 y figura 6). Un bloque de fibra de vidrio envuelto con una matriz de resina compuesto prefabricado (Fiber CAD post\&core, Angelus, Brasil) se fresó para desarrollar el poste y el núcleo (figura 7 


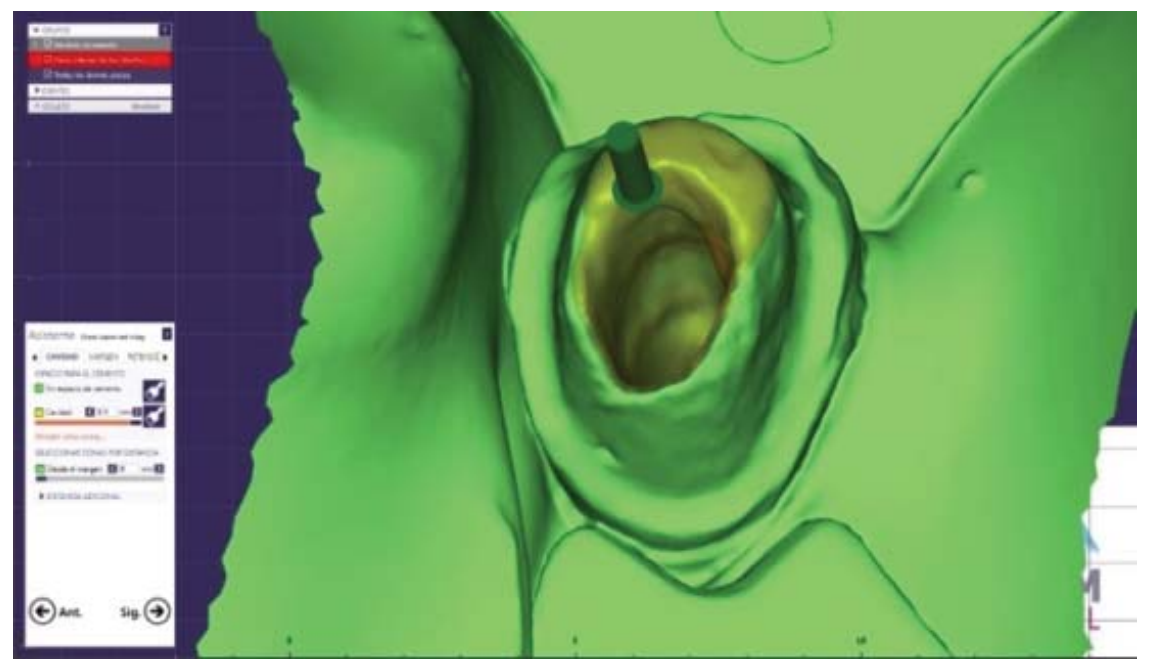

Figura 5

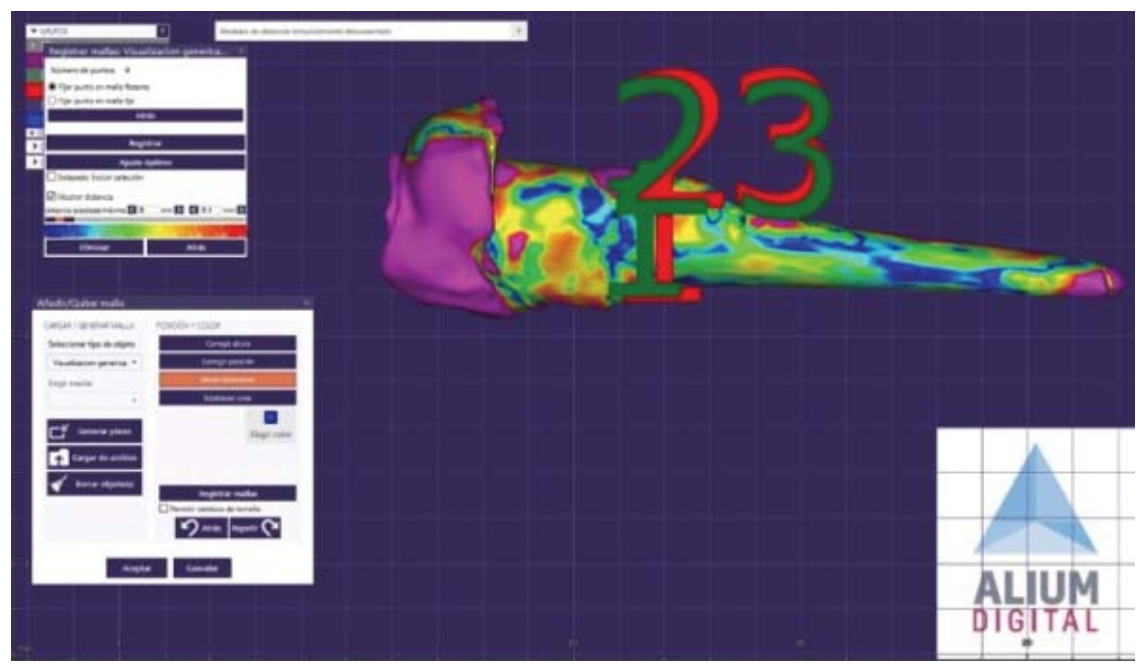

Figura 6

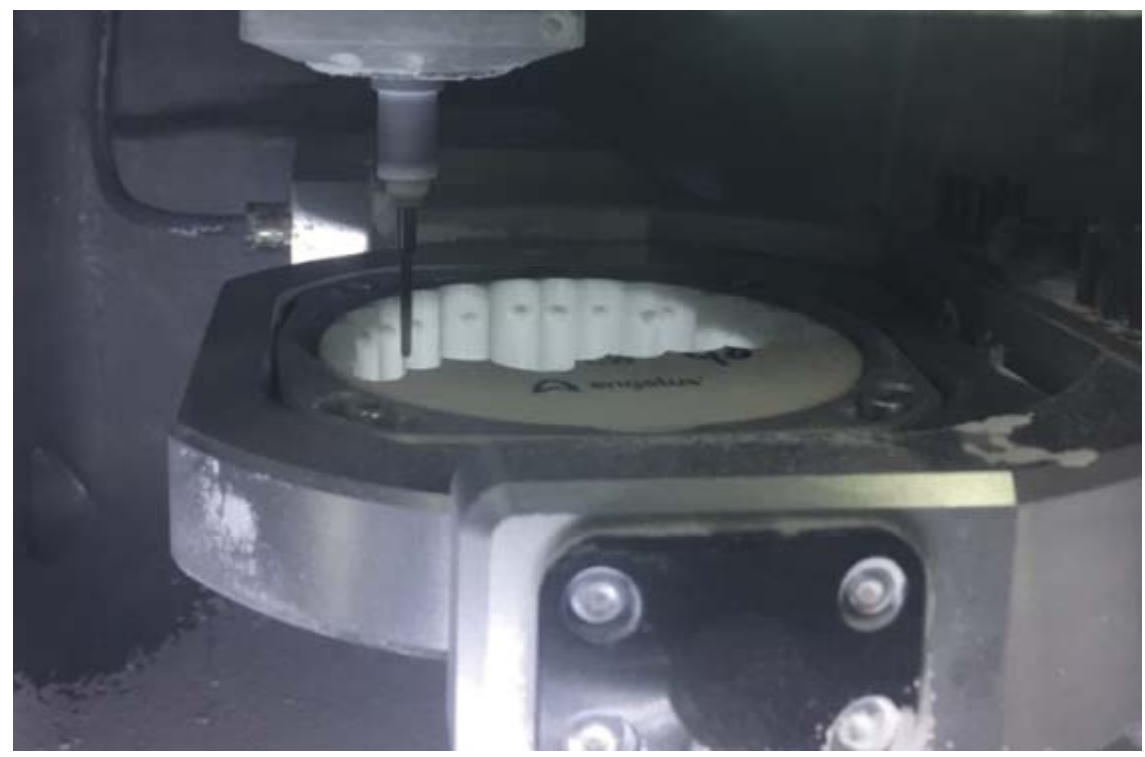

Figura 7 


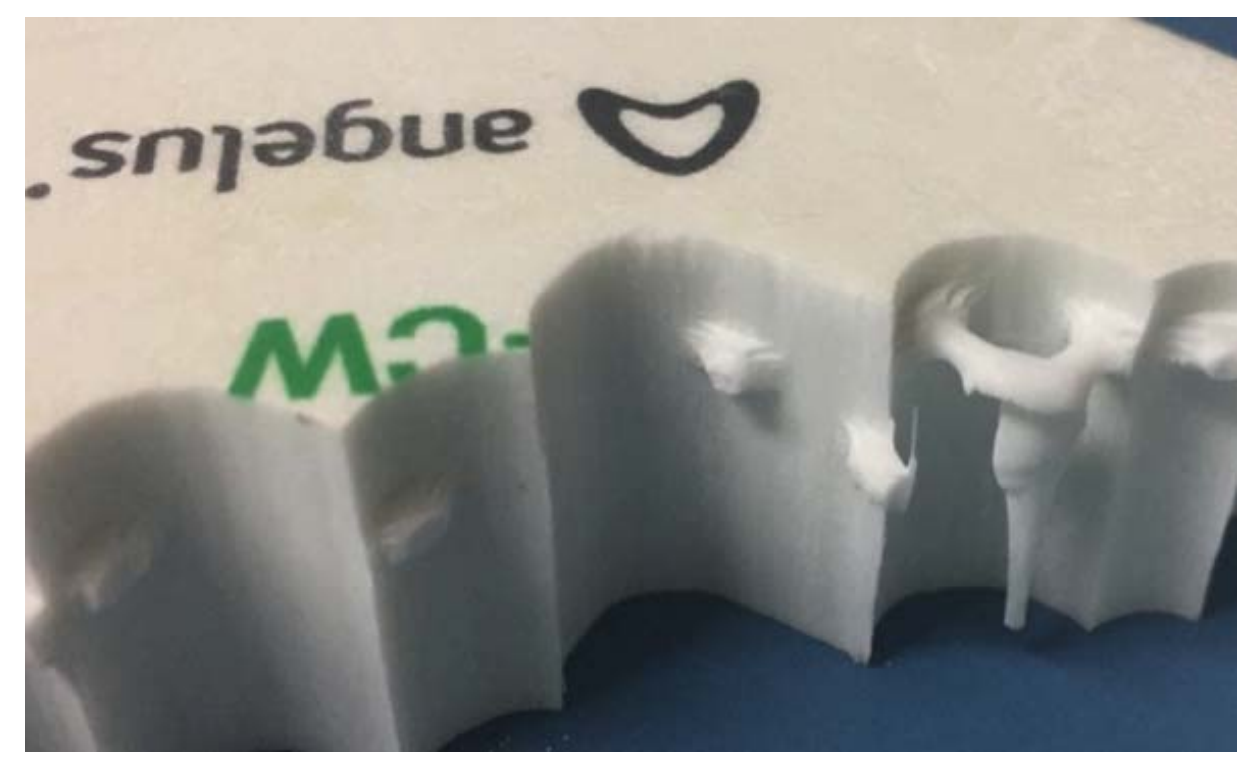

Figura 8

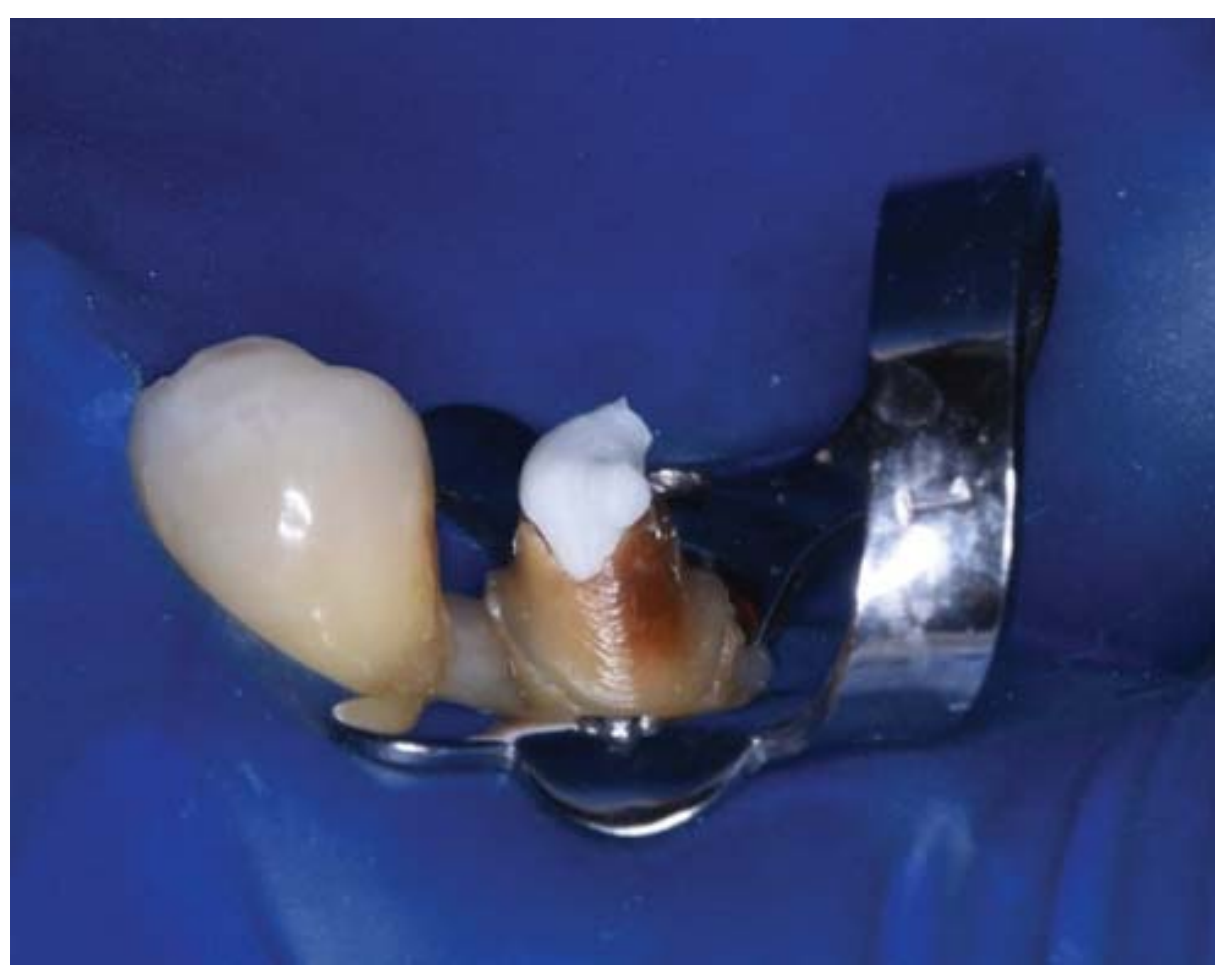

Figura 9

y figura 8), con una máquina de fresado (vhf CAM 5-S1 Impression; VHF, Ammerbuch, Alemania).

Después del fresado, se hizo la prueba del poste de fibra evidenciándose un adecuado ajuste al diente (figura 9). Se hizo la comparación con postes prefabricados para evaluar las formas (figura 10). El conducto radicular se limpió con cloruro de sodio al $0.9 \%$, se lavó, se secó, se acondicionó con ácido fosfórico al 37\% durante 10 segundos (Scotchbond, 3M, USA) (figura 10), se lavó, se secó con conos de papel \#60 (Endomedic, Sure endo Corporation, Korea). El poste de fibra cad/cam se acondicionó en hipoclorito de sodio $5 \%$ por 60 segundos, se lavó, se secó con aire y se aplicó un agente de unión en su superficie durante 60 segundos (Silano-Agente 


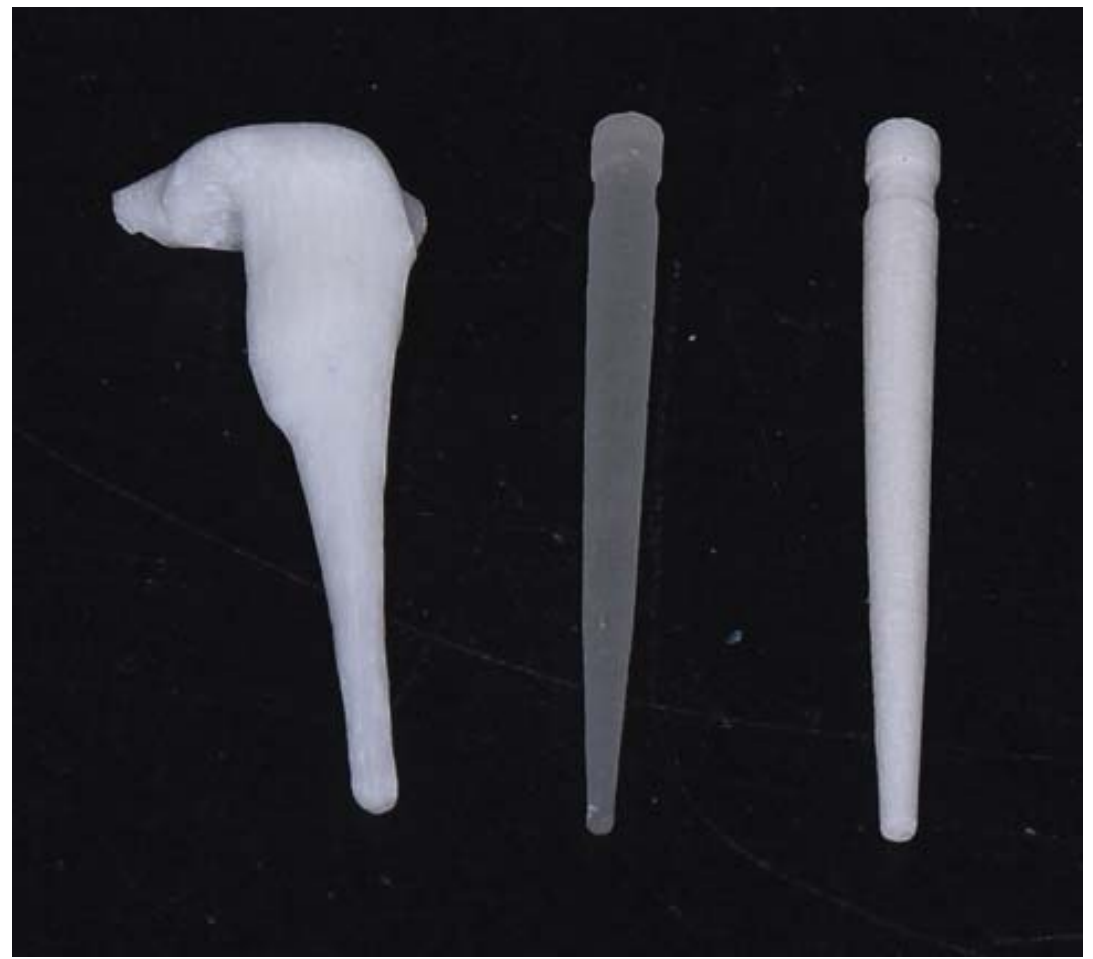

Figura 10

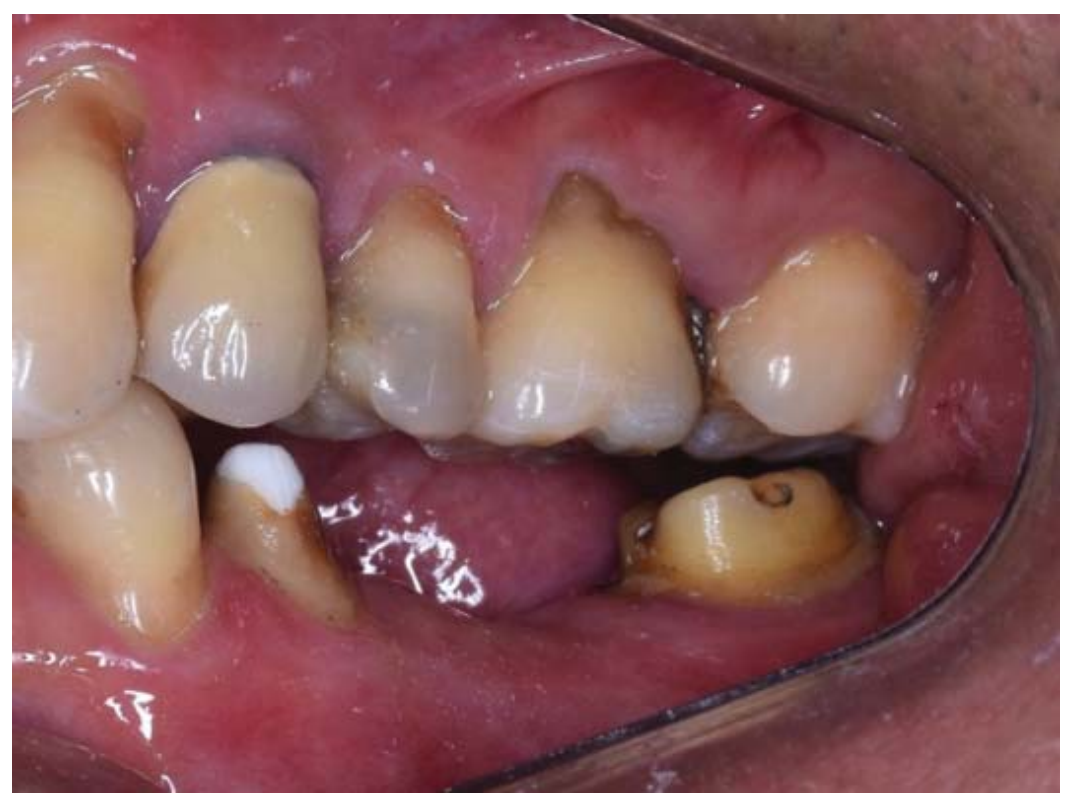

Figura 11

de Unión, Angelus, Brasil). El espigo de fibra cad/ cam se cementó al conducto usando un cemento resinoso dual autoadhesivo (RelyX-U200, 3m, USA) de acuerdo a las especificaciones del fabricante. Se realizó la polimerización del espigo durante 60 segundos con una lámpara de fotopolimerización de alta intensidad (Elipar Deep Cure, 3M, USA). Luego se realizó el tallado del pilar para un puente de tres unidades con zirconio monolítico (figura 11). Una radiografía periapical de control demostró que el poste y el núcleo de la fibra se encontraron bien adaptados al espacio del espigo (figura 12). Posterior a ello se realizó la cementación del puente de óxido de zirconio monolítico en las piezas 35,36 y37 (figura 13). 


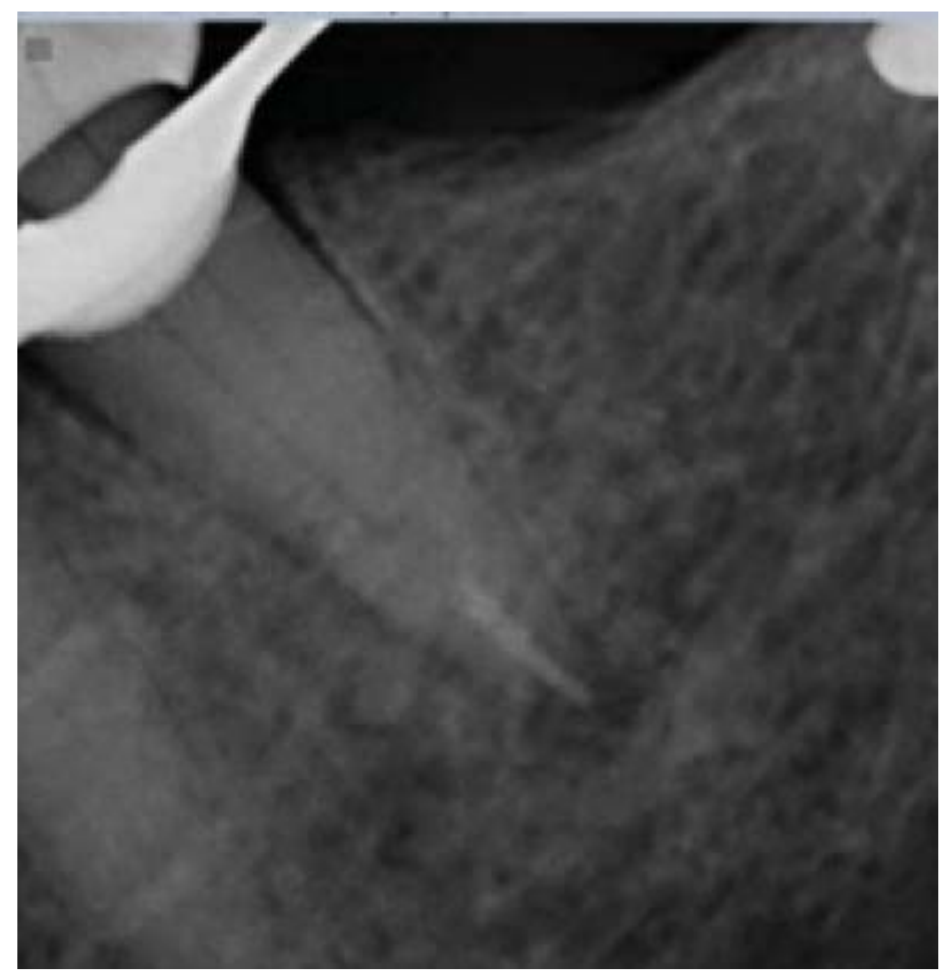

Figura 12

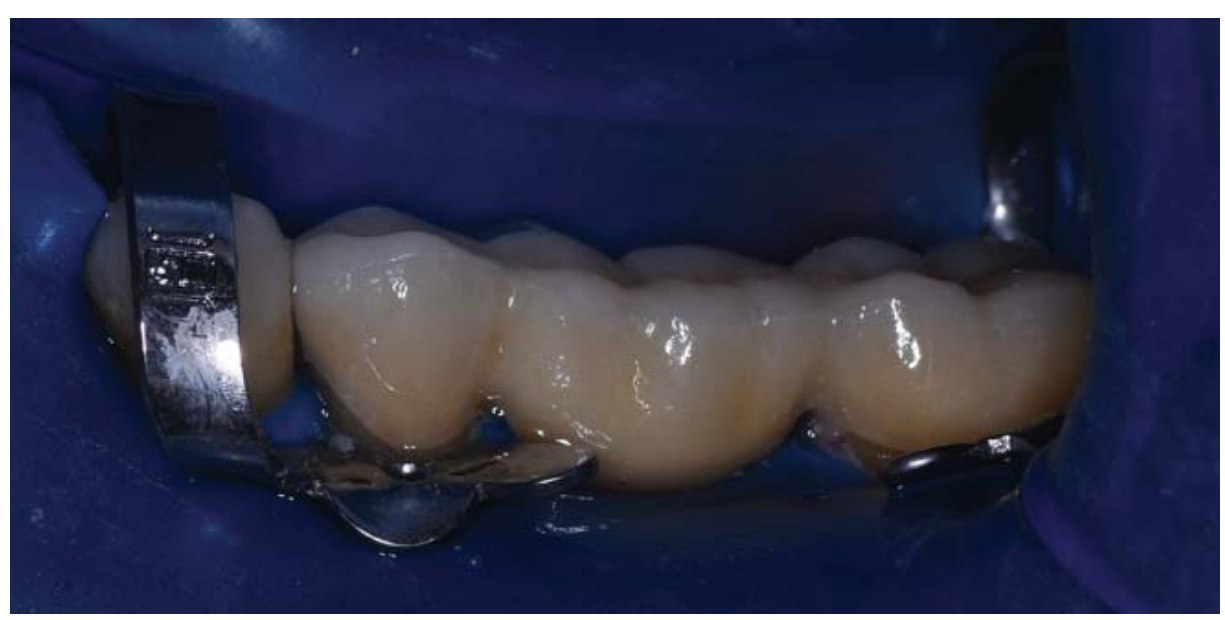

Figura 13

\section{DISCUSIÓN}

En la actualidad, y gracias a la evolución de la odontología adhesiva y materiales cerámicos, los materiales compuestos reforzados con fibra de vidrio han aumentado sus usos clínicos. Para casos de conductos radiculares amplios y necesidad de reconstrucción del muñón coronal, se requiere reforzar los espigos de fibra prefabricados con una base de resina compuesta (6).
El reporte de caso demuestra que con la ayuda de la tecnología CAD/CAM se puede crear un sistema de espigo y muñón coronal monolítico, que puede adaptarse bien y reducir el grosor de la capa de cemento.

El módulo de elasticidad del espigo y muñón CAD/CAM es similar al de la dentina $(3,4)$, lo que permite disipar mejor el estrés en el conducto radicular debilitado. El espigo y muñón CAD/CAM 
es más grueso comparados con el poste de fibra pre fabricados, lo cual se traduciría en una mayor resistencia a las fuerzas masticatorias. Según últimos estudios in vitro, en comparación con los métodos convencionales, la restauración con postes de fibra de vidrio CAD/CAM puede aumentar la resistencia general a la fractura de la raíz y reducir la aparición de fracturas irreparables $(15,16)$.

Se debe tener en cuenta las propiedades ópticas del espigo de fibra de vidrio CAD/CAM, donde el color del sustrato permite la confección de coronas libres de metal más estéticas. Las principales desventajas del poste fibra de vidrio CAD / CAM son su proceso de producción relativamente complejo y que requiere 2 sesiones de tratamiento (a menos que se cuente con dichos equipos en el ambiente clínico) $(17,18)$.

Se observó una excelente adaptación del poste de fibra CAD/CAM diseñada a partir de una imagen digital de la impresión realizada con una doble de impresión con silicona de adición; este resultados guarda relación con los resultados obtenidos por diversos estudios que indican mayor precisión y confiabilidad en el escaneo indirectos a través de los modelos de yeso o las impresiones $(19,20)$.

\section{Correspondencia}

\section{Henry Vilcapoma Guerra}

Correo electrónico: henry.vilcapoma@upch.pe

\section{REFERENCIAS BIBLIOGRÁFICAS}

1. Ferrari M, Cagidiaco MC, Grandini S, De Sanctis M, Goracci C. Post placement affects survival of endodontically treated premolars. J Dent Res. 2007;86(8):729-34.

2. Zadik Y, Sandler V, Bechor R, Salehrabi R. Analysis of factors related to extraction of endodontically treated teeth. Oral Surg Oral Med Oral Pathol Oral Radiol Endod. 2008;106(5):e31-5.

3. Torres-Sánchez C, Montoya-Salazar V, Córdoba P, et al. Fracture resistance of endodontically treated teeth restored with glass fiber reinforced posts and cast gold post and cores cemented with three cements. J Prosthet Dent. 2013;110(2):127-33.

4. Sadek F, Monticelli F, Goracci C, Tay FR, Cardoso PE, Ferrari M. Bond strength performance of different resin composites used as core materials around fiber posts. Dent Mater. 2007;23:95-9.
5. Cagidiaco MC, Radovic I, Simonetti M, Tay F, Ferrari M. Clinical performance of fiber post restorations in endodontically treated teeth: 2-year results. Int J Prosthodont. 2007;20(3):293-8.

6. Peters OA. Current challenges and concepts in the preparation of root canal systems: a review. J Endod. 2004;30(8):559-67.

7. Coniglio I, Garcia-Godoy F, Magni E, Carvalho CA, Ferrari M. Resin cement thickness in oval-shaped canals: oval vs. circular fiber posts in combination with different tips/drills for post space preparation. Am J Dent. 2009;22(5):290-4.

8. Mirmohammadi H, Gerges E, Salameh Z, Wesselink PR. Effect of post diameter and cement thickness on bond strength of fiber posts. Quintessence Int. 2013;;44(10):801-10. doi: 10.3290/j.qi.a30179

9. Gomes GM, Rezende EC, Gomes OM, Gomes JC, Loguercio AD, Reis A. Influence of the resin cement thickness on bond strength and gap formation of fiber posts bonded to root dentin. J Adhes Dent. 2014;16(1):71-8

10. Singh A, Logani A, Shah N. An ex vivo comparative study on the retention of custom and prefabricated posts. J Conserv Dent. 2012;15(2):183-6.

11. Grandini S, Sapio S, Simonetti M. Use of anatomic post and core for reconstructing an endodontically treated tooth: a case report. JAdhes Dent. 2003;5(3):2437.

12. Porciani PF, Coniglio I, Magni E, Grandini S. Fiber post fitting to canal anatomy: a review of the morphology and shape of root canal system. Int Dent SA. 2008; 10: 52-58.

13. Grande NM, Butti A, Plotino G, Somma F. Adapting fiber-reinforced composite root canal posts for use in noncircular-shaped canals. Pract Proced Aesthet Dent. 2006; 18: 593-599.

14. Plotino G, Grande NM, Pameijer CH, Somma F. Influence of surface remodeling using burs on the macro and micro surface morphology of anatomically formed fiber posts. Int Endod J. 2008; 41: 345-355.

15. Borzangy SS, Saker SM, Al-Zordk WA. Effect of restoration technique on resistance to fracture of endodontically treated anterior teeth with flared root canals. J Biomed Res. 2019; 33(2): 131-138.

16. Pang J, Feng C, Zhu X, et al. Fracture behaviors of maxillary central incisors with flared root canals restored with CAD/CAM integrated glass fiber postand-core. Dent Mater J. 2019;38(1):114-119.

17. Liu P, Deng XL, Wang XZ. Use of a CAD/CAM fabricated glass fiber post and core to restore fractured anterior teeth: a clinical report. J Prosthet Dent. 2010; 103: 330-333.

18. Chen Z, Li Y, Deng X, Wang X. A novel computeraided method to fabricate a custom one-piece glass fiber dowel-and-core based on digitized impression and 
crown preparation data. J Prosthodont. 2014; 23: 276-283. 19. Boldt F, Weinzierl C, Hertrich K, Hirschfelder U. Comparison of the spatial landmark scatter of various 3D digitalization methods. J Orofac Orthop. 2009; 70: 247-263.
20. Flügge TV, Schlager S, Nelson K, Nahles S, Metzger MC. Precision of intraoral digital dental impressions with iTero and extraoral digitization with the iTero and a model scanner. Am J Orthod Dentofacial Orthop. 2013; 144: 471-478.

Recibido: 15-02-2019

Aceptado: 14-05-2019 